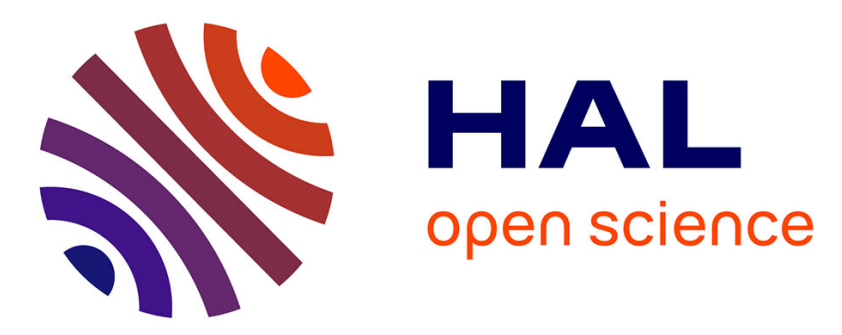

\title{
Vocal tract resonances in singing: Strategies used by sopranos, altos, tenors, and baritones
}

\author{
Nathalie Henrich Bernardoni, John Smith, Joe Wolfe
}

\section{To cite this version:}

Nathalie Henrich Bernardoni, John Smith, Joe Wolfe. Vocal tract resonances in singing: Strategies used by sopranos, altos, tenors, and baritones. Journal of the Acoustical Society of America, 2011, 129 (2), pp.1024-1035. 10.1121/1.3518766 . hal-00569451

\section{HAL Id: hal-00569451 \\ https://hal.science/hal-00569451}

Submitted on 25 Aug 2011

HAL is a multi-disciplinary open access archive for the deposit and dissemination of scientific research documents, whether they are published or not. The documents may come from teaching and research institutions in France or abroad, or from public or private research centers.
L'archive ouverte pluridisciplinaire HAL, est destinée au dépôt et à la diffusion de documents scientifiques de niveau recherche, publiés ou non, émanant des établissements d'enseignement et de recherche français ou étrangers, des laboratoires publics ou privés. 


\title{
Vocal tract resonances in singing: Strategies used by sopranos, altos, tenors, and baritones
}

\author{
Nathalie Henrich \\ Department of Speech and Cognition, GIPSA-lab (UMR5216: CNRS, INPG, University Stendhal, UJF), \\ Grenoble, France \\ John Smith and Joe Wolfe \\ School of Physics, University of New South Wales, Sydney, New South Wales 2052, Australia
}

(Received 13 July 2010; revised 21 October 2010; accepted 27 October 2010)

\begin{abstract}
The first two vocal tract resonances $(R 1$ and $R 2)$ of 22 classically trained sopranos, altos, tenors, and baritones were measured while they sang four different vowels over their normal pitch range. The resonances of the tract and the harmonics of the voice were measured simultaneously by injecting a broadband acoustic current into the tract at their mouth. Sopranos were found to tune $R 1$ close to the fundamental frequency $f_{0}\left(R 1: f_{0}\right.$ tuning) over at least part of their upper range for all vowels studied, particularly when $f_{0}$ was around or above the value of $R 1$ for speech. Additionally, most sopranos employed $R 2: 2 f_{0}$ tuning over some of their range, often simultaneously with $R 1: f_{0}$ tuning. Altos used $R 1: f_{0}$ tuning for vowels having lower values of $R 1$ in speech, but can switch to $R 1: 2 f_{0}$ tuning in the lower part of their range. Tenors and baritones generally used $R 1: 2 f_{0}$ and $R 1: 3 f_{0}$ tunings over part of their range and employed a number of different tunings to higher harmonics at lower pitch. These results indicate that singers can repeatedly tune their resonances with precision, and that there can be considerable differences in the resonance strategies used by individual singers, particularly for voices in the lower ranges. (C) 2011 Acoustical Society of America. [DOI: 10.1121/1.3518766]
\end{abstract}

PACS number(s): 43.75.Rs [ADP]

Pages: 1024-1035

\section{INTRODUCTION}

The frequencies of the first two or three resonances of the vocal tract may be varied by movements of articulators, such as tongue, jaw, lips, and larynx. Each resonance, with frequency $R 1, R 2$, etc., usually produces a maximum in the envelope of the spectrum of the voice. In speech, these spectral maxima ${ }^{1}$ have roles in characterizing vowels and some consonants (Fant, 1970; Stevens, 2000; Clark et al., 2007). The vocal tract resonances not only continue to perform these functions when singing text, but can also have important additional functions. These resonances can enhance the overall sound level of the voice by improving the coupling between the glottis and the external radiation field. The vibrating vocal folds produce a signal with fundamental frequency $f_{0}$, which is usually unrelated to the $R \mathrm{i}$. When a harmonic of the voice (an integral multiple of $f_{0}$ ) lies sufficiently close to any one of the $R \mathrm{i}$, that harmonic is radiated strongly. Further, it has been suggested that if $f_{0}$ occurs at a frequency slightly below that of a resonance, the inertive load on the vocal folds may enhance their vibration and stability (Titze, 1988, 2008). Maintaining a high sound level is important to many classical singers, who often perform unamplified in large auditoria, sometimes accompanied by large orchestras. Resonance tuning, i.e., the adjustment of the frequency of a resonance to match that of a harmonic of the voice, thus offers singers a technique that is believed to increase loudness with little extra vocal effort. These adjustments also have implications for speech and singing synthesis. This paper investigates whether singers in different vocal ranges tune $R 1$ and/or $R 2$ to the fundamental and/or other harmonics of the voice.
The possibility of implementing resonance tuning necessarily requires a suitable overlap in frequency between the possible range of a resonance and a harmonic of the voice. Figure 1 illustrates various possible tuning strategies, including those that have been measured and/or proposed in the literature for four voice types: Soprano, alto, tenor, and bass. The significant differences in possible strategies between these vocal ranges are now discussed.

\section{A. Soprano and alto ranges}

Figure 1(a) illustrates that the nominal range of the soprano voice, about C4 to C6, (261-1046 Hz) roughly coincides with the range of $R 1$ in speech. This raises the possibility that sopranos might tune $R 1$ to the fundamental frequency $f_{0}$, as concluded by Sundberg et al. (Sundberg, 1975, 1987; Sundberg and Skoog, 1997). Later studies confirmed that classically trained sopranos do indeed raise $R 1$ during singing over the higher part of their range $\left(500<f_{0}<1000 \mathrm{~Hz}\right)$, so that it is approximately the same as $f_{0}$ (Joliveau et al., 2004a). This $R 1: f_{0}$ tuning is perhaps more likely in the upper octave of the soprano range, when $f_{0}$ starts to increase above the values of $R 1$ in speech.

There exist some additional possibilities for the soprano voice. Figure 1(a) indicates that $R 2: 2 f_{0}$ tuning could occur in the upper octave of the soprano range from C5 to C6. $R 1: 2 f_{0}$ tuning might also occur in the lower octave of the soprano voice (C4 to C5). Interestingly for coloratura or other sopranos who sing well above the normal range and past the upper limit of $R 1$, there is only one possible strategy, i.e., to use $R 2: f_{0}$ tuning. Recent measurements have confirmed that this strategy is indeed employed (Garnier et al., 2010). That very high range — out of reach of most sopranos — is not studied here. 

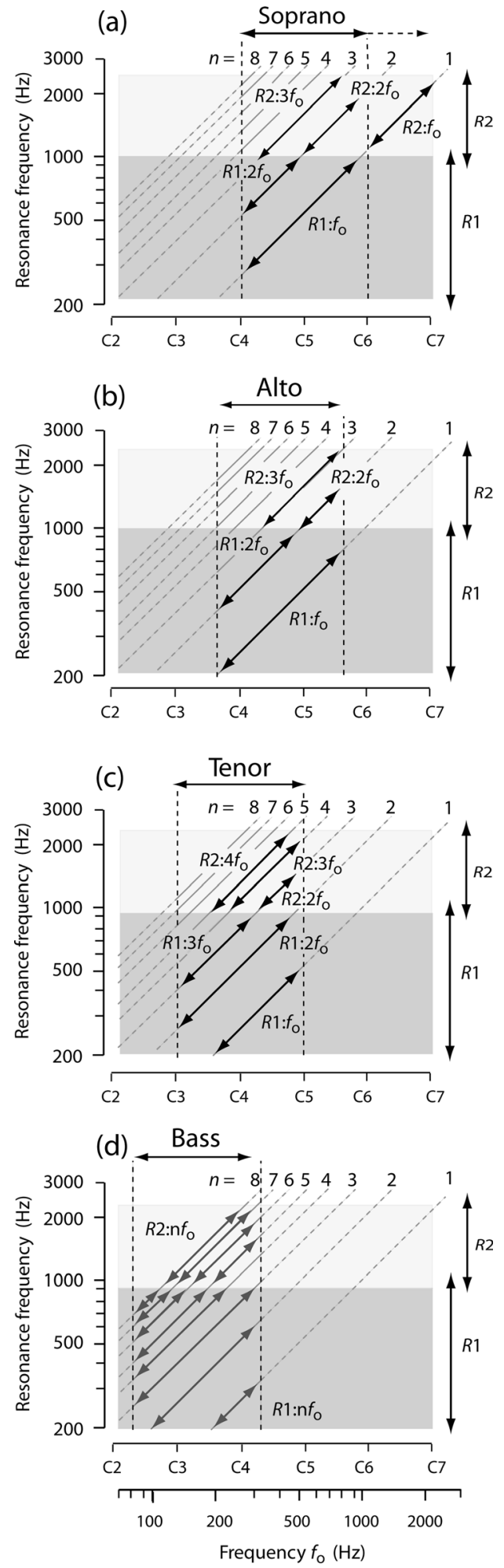

FIG. 1. A schematic showing possible resonance-tuning strategies for different voice ranges on a log-log plot. Typical ranges of the vocal tract resonances $R 1$ and $R 2$ are shown in gray. Within each voice range the diagonal, dashed gray lines indicate when a resonance frequency coincides with the $n$th harmonic $\left(n f_{0}\right)$ of the sung pitch $\left(f_{0}\right)$; i.e., the possible relationships $R \mathrm{i}=n f_{0}$ for $n=1-8$. Only the first eight harmonics are shown. The vertical dashed lines indicate the nominal limits of each singing range. Within the soprano, alto, and tenor ranges, the double-headed diagonal arrows indicate various possible tuning strategies, including those that have been measured or proposed. Gray double-headed arrows indicate some of the possibilities for the bass range. A known tuning $\left(R 2: f_{0}\right)$ is also shown for the coloratura or whistle range that lies above the normal soprano range studied in this paper (Garnier et al., 2010).
The nominal range of the alto voice (G3 to G5) has considerable overlap with the soprano range and altos might also be expected to utilize similar tuning strategies, e.g., $R 1: f_{0}$ tuning might be expected, particularly over the higher part of their range. Figure 1(b) shows that $R 1: 2 f_{0}$ tuning is also a possible strategy, particularly in the lower range. Indeed it has been proposed that $R 1: 2 f_{0}$ tuning is employed in the "belting" style of singing (Schutte and Miller, 1993). More recently $R 1: 2 f_{0}$ tuning over the range $300<f_{0}<500$ has been found in a traditional Bulgarian style of singing, which produces a voice of unusual timbre and surprising loudness (Henrich et al., 2007). The possibility of $R 2: 2 f_{0}$ tuning, or perhaps even $R 2: 3 f_{0}$ tuning, exists in the upper region of the alto range.

\section{B. Tenor, baritone, and bass ranges}

The lower fundamental frequency of the male voice range offers a much wider range of strategies. In the lowest range of men's voices $\left(f_{0} \sim 100 \mathrm{~Hz}\right)$, systematic resonance tuning would seem to offer little advantage; the harmonics are closely spaced and at least one will usually fall sufficiently close to any normal value of $R 1$ to obtain some useful boost in sound level. Indeed Fig. 1(d) indicates that six or seven harmonics fall within the normal range of $R 1$ in the lower octave - one is likely to be a useful match for any note-pitch combination, with little adjustment. The values of $R 1$ might then be similar to those of speech, although small shifts in resonances might still be advantageous to enhance further the sound level. Similarly there are also many possibilities for matching $R 2$ with harmonics in this region.

For baritones and tenors, however, deliberate tuning might have advantages. Near the upper end of the tenor range (nominally about C5 at $523 \mathrm{~Hz}$ ), the harmonic spacing approaches $500 \mathrm{~Hz}$ and consequently it is then possible that no harmonic might be nearer than $250 \mathrm{~Hz}$ to the value of $R 1$ for a vowel in speech. For the vowel $/ \mathrm{u} /$, the typical values of $R 1$ are low and fall well within the normal singing $f_{0}$ range of baritones, and so, for this vowel especially, one might expect some baritones and tenors to take advantage of $R 1: f_{0}$ tuning. Figure 1 (c) shows that $R 1: 2 f_{0}$ tuning is also possible over the full tenor range, and that $R 1: 3 f_{0}$ tuning might be useful in the lower part of the range. There are also several possibilities of tuning $R 2$, including $R 2: 2 f_{0}, R 2: 3 f_{0}$, and $R 2: 4 f_{0}$ tuning.

Strong evidence for $R 1: f_{0}$ tuning in tenors is not available. Titze et al. (1994) used an analysis-by-synthesis technique and adjusted both formant frequencies and glottal parameters of a linear source-filter model to match the spectra of a sample of tenor voices. The frequency of the first resonance was found well above the fundamental for all vowels except /u/. They interpreted the absence of tuning $R 1$ to $f_{0}$ in tenor voices as due to a desire to maintain a characteristic male quality. Later Tom and Titze (2001), also using synthesis, reported that the tenor in that study appeared to tune $R 1$ to $f_{0}$ in two out of nine possibilities for the vowel /a/.

However, there is indirect evidence for all the other possible resonance tunings discussed above for the male voice. Thus Miller and Schutte (1990) reported evidence for $R 1: 2 f_{0}$ and $R 2: 2 f_{0}$ tuning on isolated notes of baritones via measurements of sub- and supra-glottal pressure. There is also evidence 
suggesting $R 1: 2 f_{0}$ tuning for males in the ojkanje style of Croatian singing (Boersma and Kovacic, 2006). Schutte et al. (2005) suggested that $R 2: 3 f_{0}$ tuning occurs on the high Bb4 notes on recordings of tenor(s). Neumann et al. (2005) inferred from the sound spectrum that, for male opera singers in the "chest" register, the second harmonic was "resonated by" the first resonance ( $R 1: 2 f_{0}$ tuning) and the fourth harmonic by $R 2$ ( $R 2: 4 f_{0}$ tuning) with the implication that then $R 2 \approx 2 R 1 \approx 4 f_{0}$. Across the passaggio, $R 2$ often fell near $3 f_{0}$.

To investigate resonance tuning reliably, it is important that the frequencies of the resonance and the relevant harmonic should be determined precisely and independently (Joliveau et al., 2004a). For example, studies of singing at high pitch that use only the sound and determine the resonance frequencies from the voice harmonics are inherently quite inaccurate (Monsen and Engebretson, 1983) and are further complicated by the frequency dependence of the glottal source.

Because most previous studies used indirect or imprecise methods to deduce or to estimate resonance frequencies, this paper takes a different approach that involves injecting a synthetic broadband acoustic signal at the singer's mouth during singing. A microphone records both the singing and the response of the vocal tract to this broadband excitation. For this study, only singers trained in the classical tradition were investigated; they were volunteers with experience ranging from amateur to professional.

\section{MATERIALS AND METHODS}

\section{A. Resonance measurements}

The measurements were conducted at UNSW in a room treated to reduce reverberation and to reduce external noise. The technique used for resonance measurement has been described previously (Epps et al., 1997; Joliveau et al., 2004b). Briefly, a small source of broadband acoustic current and a microphone are positioned adjacent to each other on a flexible mounting so that they just touch the subject's lower lip throughout the experiment. This does not affect the ability of singers to open the mouth or to move the jaw. A computer (Macintosh IIci, Apple Computer, CA) synthesizes the broadband signal as a sum of sine waves with frequencies spaced at $5.38 \mathrm{~Hz}$ and phases adjusted to improve the signal to noise ratio (Smith, 1995). During an initial calibration procedure, the microphone measures $p_{\text {closed, }}$, the pressure spectrum in response to the broadband current with the mouth closed. Subsequently the microphone measures $p_{\text {open, }}$, the spectrum of the response with the mouth open and in parallel with the radiation impedance at the mouth, and the ratio $\gamma$ is calculated, where $\gamma$ is given by

$$
\gamma=p_{\text {open }} / p_{\text {closed }} \text {. }
$$

Because the broadband source is a good approximation to a current source, $\gamma$ is effectively equal to the ratio of the impedance of the tract at the mouth, in parallel with the radiation field, to that of the radiation field alone. The resonance frequencies were manually detected from the recorded data by one author and checked by another. In some measurements, particularly for closed vowels, the impedance of the radiation field dominates the measurements to such an extent that the tract resonances cannot be unequivocally and precisely identified. This occurred in $16 \%$ of the 1374 measurements of $R 1$, and in $6 \%$ of the measurements of $R 2$. In these cases that particular datum was disregarded. The error in resonance detection is typically $\pm 11 \mathrm{~Hz}$.

\section{B. The subjects}

Twenty-two subjects volunteered to take part. Their experience varied from nationally recognized to amateur singers. Their range and experience are given in Table I. All but four described their singing style as Western classical, although some had had experience in other styles. The other styles were jazz (singer B3) and musical theatre (singers S3, T3). Singer T7 had no defined style. Baritones were used rather than basses because Fig. 1(d) indicates that deliberate resonance tuning would be very difficult to detect at very low pitch. Sopranos had been studied earlier in our laboratory (Joliveau et al., 2004a,b). However, in that study the singers had been asked to sing softly, because of limited power in the injected sound signal. The apparatus used here had higher power so it was judged worthwhile to conduct new measurements on the soprano range at a louder singing level.

\section{The protocol}

This study used the same set of four vowels as an earlier study (Joliveau et al., 2004a,b), which had been chosen to ensure ease of singing and measurement, sampling of the phoneme space, and the effects of lip rounding. Each word

TABLE I. Details of the experimental subjects. Experience is specified using the taxonomy of Bunch and Chapman (2000): 3 = national/big city, $4=$ regional/touring, $5=$ local community (often semi-professional), 7 = full-time voice student, $8=$ amateur (sings for pleasure). The lowest and highest pitches lie within the comfortable range of each singer.

\begin{tabular}{|c|c|c|c|c|c|c|c|c|c|c|c|c|c|}
\hline \multicolumn{6}{|c|}{ Baritones } & \multicolumn{8}{|c|}{ Tenors } \\
\hline Singer & B1 & B2 & B3 & $\mathrm{B}$ & & $\mathrm{T} 1$ & $\mathrm{~T} 2$ & $\mathrm{~T} 3$ & $\mathrm{~T} 4$ & T5 & T6 & $\mathrm{T} 7$ & $\mathrm{~T} 8$ \\
\hline Taxonomy & 7 & 5 & 3 & 8 & & 4 & 3 & 4 & 8 & 3 & 3 & 8 & 5 \\
\hline Lowest pitch & $\mathrm{G} 2$ & $\mathrm{G} 2$ & A2 & $\mathrm{F}$ & & G2 & $\mathrm{A} 2$ & C3 & C3 & C3 & $\mathrm{F} 2$ & $\mathrm{C} 3$ & A2 \\
\hline Highest pitch & $\mathrm{F} 4$ & $\mathrm{~F} 4$ & D4 & A & & A4 & A4 & C5 & C5 & B4 & D5 & G4 & A4 \\
\hline \multicolumn{7}{|c|}{ Altos } & \multicolumn{7}{|c|}{ Sopranos } \\
\hline Singer & A1 & A2 & & A3 & A4 & & $\mathrm{S} 1$ & $\mathrm{~S} 2$ & S3 & & S4 & S5 & S6 \\
\hline Taxonomy & 8 & 8 & & 8 & 8 & & 3 & 8 & 4 & & 8 & 8 & 7 \\
\hline Lowest pitch & E3 & G3 & & F3 & D3 & & G3 & G3 & G3 & & B3 & G3 & $\mathrm{C} 4$ \\
\hline Highest pitch & G5 & D5 & & E5 & C5 & & D6 & B5 & A5 & & C6 & B5 & G6 \\
\hline
\end{tabular}


to be sung was presented in writing on a card using the form $h<$ vowel $>d$, i.e., hard, hoard, who'd, and heard.

Subjects were asked to sing each note, at a comfortable level and with as little vibrato as possible, in their trained singing style, at a pitch that was indicated by a glockenspiel. For each vowel, each note was presented in an ascending scale, starting at the lowest note of what each singer identified as the comfortable range of his or her singing voice and continuing to the highest note in the comfortable range. For each vowel, after each scale had been completed, the subjects were asked twice to repeat two or more of the notes in the comfortable range at a similar loudness. This was to allow an estimate of the reproducibility. Following this task, they were asked to speak the carrier word rather than sing it.

For each token, the broadband signal was started after the singer had begun a note and they were instructed to continue for a second or so after it finished. Thus, for each token, the recording included a sample of voice alone, voice plus broadband signal followed by voice alone.

\section{RESULTS AND DISCUSSION}

\section{A. The measured vocal tract resonances}

\section{The resonances of speech}

Table II shows that the average measured values of $R 1$ and $R 2$ for speech were lower for altos than for sopranos in all cases, except for $R 2$ in "who'd." However, in our small sample only the values for $R 1$ in "heard" and "hoard" and $R 2$ in "hard" and "hoard" differed significantly at the $5 \%$ level between altos and sopranos. There were no statistically significant differences between the resonances of tenors and baritones. Thus in our sample, the voice range classifications of alto and soprano, and tenor and baritone, were not correlated with their values of $R 1$ and $R 2$ for speech. This differs from the results of Cleveland (1977) where a correlation was found between the resonance frequencies of speech and the voice range classification of male singers. Although possibly

TABLE II. The measured resonance frequencies for speech for the singers in different vocal ranges.

\begin{tabular}{lcccc}
\hline \hline & \multicolumn{4}{c}{ Vowel } \\
\cline { 2 - 5 } Voice & Hard & Heard & Hoard & Who'd \\
\hline \multirow{4}{*}{ Soprano } & $825 \pm 120$ & $610 \pm 50$ & $585 \pm 45$ & $410 \pm 80$ \\
Alto & $830 \pm 95$ & $540 \pm 20$ & $515 \pm 40$ & $350 \pm 30$ \\
Female & $825 \pm 110$ & $580 \pm 50$ & $555 \pm 55$ & $390 \pm 70$ \\
Tenor & $700 \pm 85$ & $555 \pm 90$ & $545 \pm 100$ & $350 \pm 30$ \\
Baritone & $785 \pm 120$ & $520 \pm 65$ & $495 \pm 85$ & $385 \pm 85$ \\
Male & $725 \pm 100$ & $545 \pm 80$ & $530 \pm 95$ & $365 \pm 55$ \\
& & \multicolumn{4}{c}{$R 1^{\circ}(\mathrm{Hz})$} & \\
Soprano & $1340 \pm 115$ & $1560 \pm 345$ & $1065 \pm 90$ & $1340 \pm 475$ \\
Alto & $1160 \pm 60$ & $1525 \pm 140$ & $825 \pm 85$ & $1510 \pm 280$ \\
Female & $1265 \pm 130$ & $1545 \pm 270$ & $970 \pm 150$ & $1410 \pm 400$ \\
Tenor & $1175 \pm 85$ & $1340 \pm 90$ & $900 \pm 115$ & $1145 \pm 245$ \\
Baritone & $1235 \pm 50$ & $1360 \pm 125$ & $880 \pm 175$ & $1205 \pm 335$ \\
Male & $1190 \pm 80$ & $1345 \pm 100$ & $890 \pm 130$ & $1165 \pm 265$ \\
\hline \hline
\end{tabular}

complicated by resonance tuning, the similarity between $R 1$ and $R 2$ of baritones and tenors was also evident during singing. The differences calculated using a total of 1277 measurements for the 51 combinations of vowel and pitch where measurements were made for both tenors and baritones were $-25 \pm 50 \mathrm{~Hz}$ for $R 1$ and $15 \pm 90 \mathrm{~Hz}$ for $R 2$.

\section{The resonances during singing}

Rather than keeping the resonances at the values used for speech, all singers were found to vary their values of $R 1$ and $R 2$ as the fundamental frequency $f_{0}$ varied while singing. The reproducibility of the resonance measurements on individual singers was tested by measuring a number of resonances three times at the same pitch whenever possible-see Table III. At low pitches (below B4), the standard deviations in $R 1$ and $R 2$, expressed as percentage, were similar for singing and speech across all singers. However at high pitches (B4 and above) the proportional variation in $R 1$ was typically reduced by around $50 \%$. This is presumably because at high pitch, singers then have a definite optimum value for each resonance, particularly during resonance tuning, whereas for speech the values of $R 1$ and $R 2$ need only fall in the expected range for the desired vowel.

A similar effect was found when the variations among singers were examined. Table IV shows that, again, the variation in $R 1$ was reduced considerably for altos and sopranos when $f_{0}$ exceeded $R 1^{\circ}$, defined as the value of $R 1$ in speech. Otherwise, the variations between singers in different ranges appear not to be significant. Figure 2 shows the combined data for altos and sopranos when singing the vowels in "hard" and "who'd." The large reduction in variability at high pitch provides strong evidence that deliberate resonance

TABLE III. The reproducibility of vocal tract configurations for individual singers. Percentage deviations in $R \mathrm{i}$ were calculated from measurements repeated at the same pitch for each vowel and singer. The standard deviation $\sigma_{R \mathrm{i}}$ was then calculated from these data across all singers in a given frequency range and finally expressed as a percentage. The numbers in brackets indicate the number of measurements. ${ }^{*}$ Because no repetitions were made for speech in this study, the standard deviations presented for speech were calculated from a separate study on 11 female speakers performed using the same apparatus in the same laboratory (Swerdlin et al., 2010).

\begin{tabular}{lccccc}
\hline \hline & \multicolumn{5}{c}{ Vowel } \\
\cline { 2 - 6 } & Hard & Heard & Hoard & Who'd & All vowels \\
\hline & & & $\sigma_{R 1}(\%)$ & & \\
Female speech* & $7.8(41)$ & $6.8(52)$ & $6.0(55)$ & $9.4(50)$ & $7.1(251)$ \\
$\begin{array}{c}\text { Female singing } \\
\text { (below B4) }\end{array}$ & $6.6(29)$ & $8.0(34)$ & $6.8(38)$ & $5.8(27)$ & $6.8(128)$ \\
$\begin{array}{c}\text { Female singing } \\
\text { (above B4) }\end{array}$ & $3.3(11)$ & $5.7(9)$ & $2.7(6)$ & $2.0(14)$ & $3.4(40)$ \\
$\begin{array}{c}\text { Male singing } \\
\text { Female speech* }\end{array}$ & $5.6(68)$ & $4.5(83)$ & $5.5(75)$ & $6.4(69)$ & $5.5(295)$ \\
$\begin{array}{c}\text { Female singing } \\
\text { (below B4) }\end{array}$ & $2.6(42)$ & $3.6(38)$ & $6.0(38)$ & $5.5(38)$ & $4.6(156)$ \\
$\begin{array}{c}\text { Female singing } \\
\text { (above B4) }\end{array}$ & $1.1(15)$ & $2.3(12)$ & $2.9(11)$ & $9.6(14)$ & $5.2(52)$ \\
\begin{tabular}{c} 
Male singing \\
\hline \hline
\end{tabular} & $3.8(76)$ & $6.5(86)$ & $3.7(90)$ & $5.5(74)$ & $5.0(326)$ \\
\hline \hline
\end{tabular}


TABLE IV. The variation in vocal tract resonances among different singers. The standard deviations in $R \mathrm{i}, \sigma_{R \mathrm{i}}$, were calculated from measurements at the same pitch for each vowel and singer. Equivalent values for speech are given in Table II.

\begin{tabular}{lrrrr}
\hline \hline & \multicolumn{4}{c}{ Vowel } \\
\cline { 2 - 5 } & Hard & Heard & Hoard & Who'd \\
& \multicolumn{4}{c}{$\sigma_{R 1}(\mathrm{~Hz})$} \\
Female $\left(f_{0} \leq R \mathrm{i}^{\mathrm{o}}\right)$ & 145 & 85 & 105 & 50 \\
Female $\left(f_{0}>R \mathrm{i}^{\mathrm{o}}\right)$ & 35 & 35 & 40 & 25 \\
Male & 105 & 90 & 75 & 65 \\
& & & $\sigma_{R 2}(\mathrm{~Hz})$ & \\
Female & 135 & 155 & 125 & 200 \\
Male & 115 & 95 & 120 & 195 \\
\hline \hline
\end{tabular}

tuning is occurring. There are several different possibilities for resonance tuning at low pitch (see Fig. 1), and in this range resonance tuning might even increase the variation among singers if different singers tune the resonances to different harmonics.

\section{The effect of resonance tuning}

Figure 3 shows examples of the measured pressure ratio $\gamma$ during singing. In each case the fundamental of the sung pitch $f_{0}$ and its harmonics are visible as spikes superimposed over the measured broadband spectrum. Resonances in the vocal tract are associated with maxima in the broadband response. Figure 3(a) shows an example where $R 1, R 2, f_{0}$, and $2 f_{0}$ all occur at different frequencies, indicating that, in this case, there was no adjustment of resonances to harmonics. Consequently these resonances make only a modest difference to the spectral envelope of the voice. Figure 3(b) shows an example where $R 1$ coincides with $2 f_{0}$ and $R 2$ coincides with $5 f_{0}\left(R 1: 2 f_{0}\right.$ and $R 2: 5 f_{0}$ tuning, respectively). As a result, the second harmonic now has greater energy than the first harmonic, and the fifth harmonic has greater energy than the fourth, as indicated by their higher spikes.

When the frequency of a resonance is determined by the pitch of the note sung, instead of or as well as by the vowel sung, vowel quality may be affected. For sopranos, the impact on vowel intelligibility can be quite significant (e.g.,
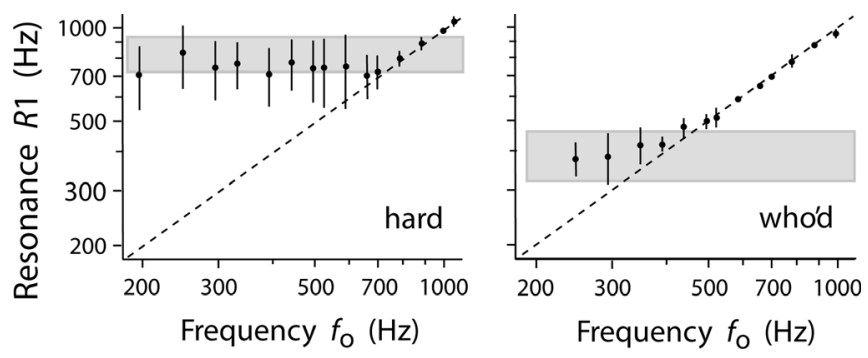

FIG. 2. The variation in resonance frequency $R 1$ among the ten female singers (altos + sopranos) as a function of frequency on a $\log -\log$ scale. The dashed diagonal line indicates the relationship $R 1=f_{0}$. The error bars indicate the standard deviations; they are too small to be shown for several points close to or on the diagonal line indicating $R 1: f_{0}$ tuning. The gray shaded areas indicate the range of \pm standard deviation of $R 1^{\circ}$ measured for these vowels and singers during speech.

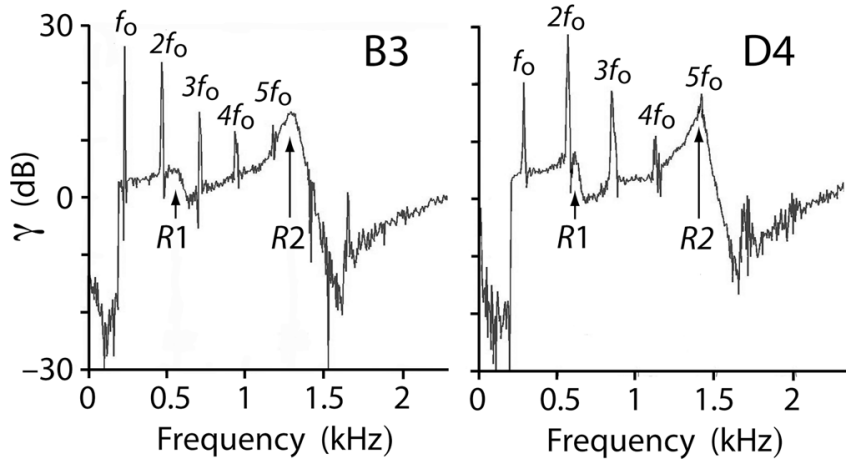

FIG. 3. The effect of matching a resonance with a harmonic for alto A1 singing the vowel in "heard" at two different pitches, mezzoforte. The quasicontinuous line shows the measured pressure ratio $\gamma\left(=p_{\text {open }} / p_{\text {closed }}\right)$ as a function of frequency; maxima in this curve indicate the resonance frequencies. The sharp peaks superposed on the curve are the harmonics of her voice. In the figure on the left she sings at pitch B3 $(247 \mathrm{~Hz})$ and none of the low harmonics fall very close to the resonances. In the figure on the right she sings at pitch D4 $(294 \mathrm{~Hz})$ and, for this note, the second and fifth harmonics fall close to the first two resonances.

Scotto di Carlo and Germain, 1985; Benolken and Swanson, 1990) as can be clearly demonstrated with appropriate sound examples (Music Acoustics, 2010). In the present study, altos, tenors, and baritones usually adjust $R 1$ over a smaller range that do sopranos, so the effects on intelligibility might be less important as the adjustments are often smaller than the characteristic separation in the vowel plane at which vowels become confused (Dowd et al., 1998). Although these resonance-tuning strategies can surely impact perceived vowel quality, they were not studied here.

\section{B. What constitutes "resonance tuning?"}

A production of any given vowel in speech will be characterized by particular values of $R 1$ and $R 2$. Figure 1 shows that, with the exception of the highest part of the soprano range, there will always be at least one value of the fundamental frequency $f_{0}$, where $f_{0}$ or one of its harmonics matches $R 1$. A similar situation occurs for $R 2$. If $R 1$ and $R 2$ were held at constant values throughout the vocal range of a

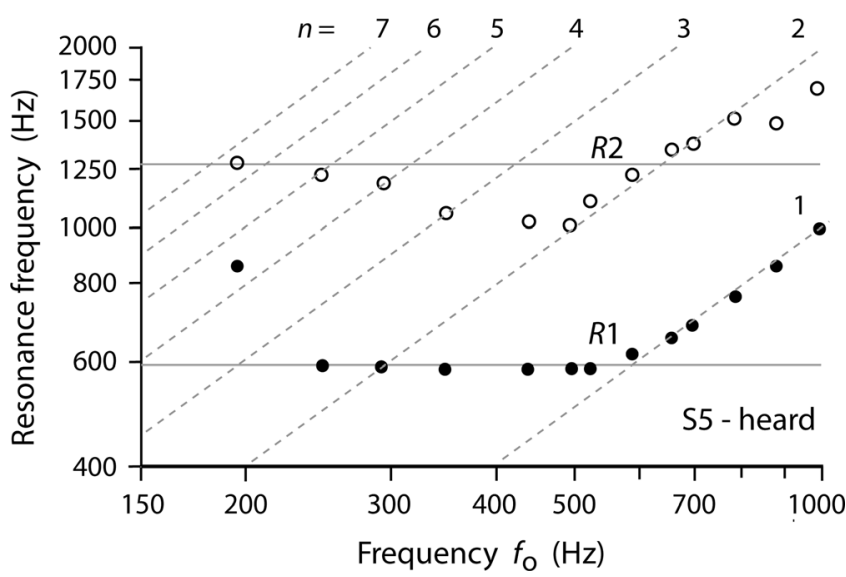

FIG. 4. An example of simultaneous $R 1: f_{0}$ and $R 2: 2 f_{0}$ tunings by soprano $\mathrm{S} 5$ on the vowel in "heard" shown in a $\log -\log$ plot. The horizontal lines indicate the values of $R 1$ and $R 2$ measured for this singer and vowel in speech. The dashed diagonal lines indicate the relationships $R \mathrm{i}=n f_{0}$. 
singer, these isolated matches would produce sudden increases in the sound level and changes in timbre as the singer sang a chromatic scale, passing $f_{0}$ through $R 1$ and $R 2$. This study is concerned with determining when resonance tuning occurs; this involves a singer adjusting the resonance frequencies to help match resonances to $f_{0}$ or a harmonic.

For the high part of the normal soprano range, only one strategy is effectively available for each resonance-resonance tuning is then "systematic" and would be expected to occur over a substantial pitch range. Figure 4 shows two examples of such

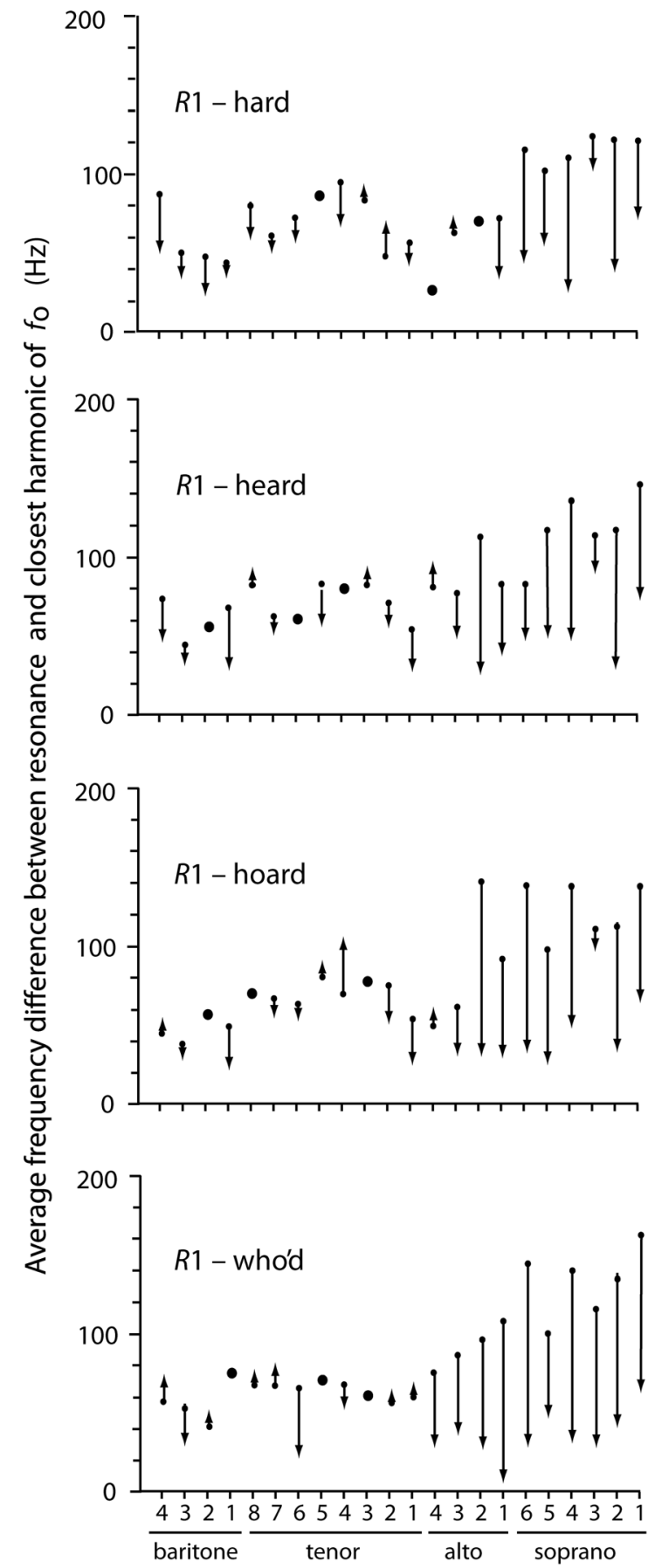

systematic resonance tuning where the same strategy is employed over a range of pitch; they are $R 1: f_{0}$ and $R 2: 2 f_{0}$ tuning.

For lower pitch ranges, the number of possibilities increases - see Fig. 1. It might therefore be expected that several different strategies for resonance tuning would be employed within a given lower voice range. Each might only occur at a couple of notes, or perhaps even at a single pitch, in which cases the term resonance tuning strategy would hardly be appropriate.

It is relatively easy to detect the presence of resonance tuning in the high voice ranges because it is likely to be
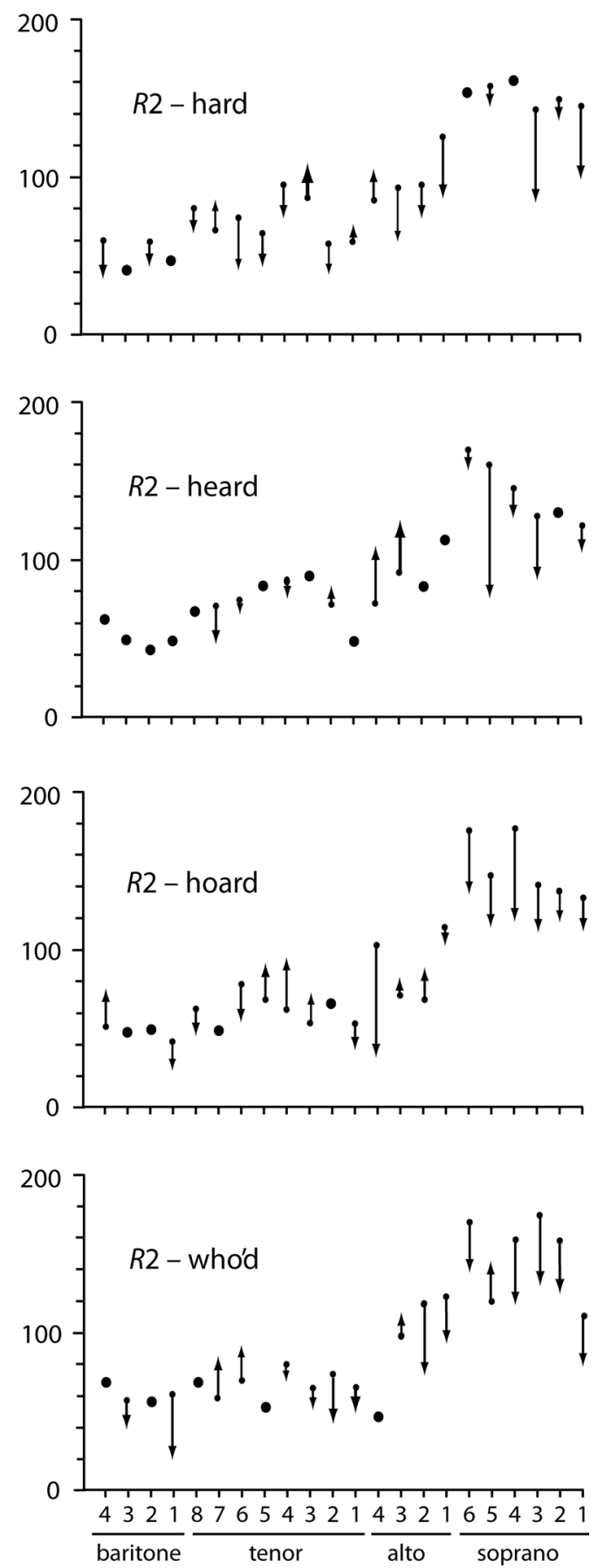

FIG. 5. The matching between resonances and harmonics for different voice ranges, the numbers on the abscissa indicating different singers. The tail of each arrow indicates the value of $\bar{\Delta}_{\mathrm{i}}^{\mathrm{o}}$, the average absolute difference in frequency between the resonance $R \mathrm{i}$ and the closest harmonic, if $R \mathrm{i}$ were held constant at its average value for that singer, vowel, and measured range. The tip of each arrow indicates $\bar{\Delta}_{\mathrm{i}}$, the average absolute difference in frequency when the measured values of $R \mathrm{i}$ are taken into account. Thus a downward pointing arrow indicates improved matching between resonance and harmonic over the measured range. A solid circle indicates that there was no significant difference between $\bar{\Delta}_{\mathrm{i}}$ and $\bar{\Delta}_{\mathrm{i}}^{\mathrm{o}}$. 
maintained over a significant range of pitch frequencies, and the tuning becomes obvious as the resonance is shifted well outside its normal range for that vowel in speech. The situation is more difficult for low-pitched voices because the resonance can be tuned, but still remain within the normal resonance range for that vowel. In order to quantify reliably the extent of resonance tuning, a parameter $\Delta_{\mathrm{i}}$ is defined that is equal to the absolute difference in frequency between the $i$ th resonance $R \mathrm{i}$ and $n f_{0}$, the harmonic closest to that resonance. Thus,

$$
\Delta_{\mathrm{i}}=\left|R \mathrm{i}\left(f_{0}\right)-n f_{0}\right|
$$

$\bar{\Delta}_{\mathrm{i}}$ can then be defined as the average value of $\Delta_{\mathrm{i}}$ over a particular pitch range.

If no attempt were made to alter $R \mathrm{i}$ and if it were maintained constant at a value denoted by $R \mathrm{i}^{\circ}$ over the pitch range of interest, this value of $\bar{\Delta}_{\mathrm{i}}$ can be defined as $\bar{\Delta}_{\mathrm{i}}^{\mathrm{o}}$, i.e.,

$$
\bar{\Delta}_{\mathrm{i}}^{\mathrm{o}}=\text { the average absolute difference }\left|R \mathrm{i}^{\mathrm{o}}-n f_{0}\right| .
$$

Thus $\bar{\Delta}_{\mathrm{i}}<\bar{\Delta}_{\mathrm{i}}^{\mathrm{o}}$ implies that the shifts of Ri with frequency are helping with the overall matching of resonances with harmonics. Figure 5 shows the values of $\bar{\Delta}_{\mathrm{i}}^{\mathrm{o}}$ (indicated by the tail of each arrow) and $\bar{\Delta}_{\mathrm{i}}$ (indicated by the tip of each arrow) calculated for each combination of singer and vowel over their measured pitch range. The values of $\bar{\Delta}_{\mathrm{i}}^{\mathrm{o}}$ were calculated using the average measured values of $R \mathrm{i}$ over the same range. Similar results were obtained using the values of $R \mathrm{i}^{\mathrm{O}}$ measured for speech.

In the absence of any resonance tuning, one would expect Fig. 5 to show about equal numbers of up- and down-pointing arrows. It is immediately apparent that the variation of $R 1$ with pitch measured for most sopranos (the exception was S3) over their pitch range dramatically reduced the average frequency difference between $R 1$ and the nearest harmonic. The matching is closest for the vowel in "who'd," which in speech has the lowest value of $R 1$ of those studied-see Table II. All sopranos and altos showed significant matching over the measured voice range for this vowel. The situation was quite different for baritones and tenors where significantly improved matching was less common; indeed on occasions the difference $\bar{\Delta}_{\mathrm{i}}^{\mathrm{o}}-\bar{\Delta}_{1}$ even changed sign indicating a larger average difference in frequency between resonance and harmonic.

The matching was more varied for $R 2$, with occasional significant improvements in matching, but also many cases where the matching became worse. It now remains to look at each voice type in detail and to determine the particular resonance strategies involved.

\section{Resonance tuning in the soprano range}

Figure 6 shows the ranges over which $R 1$ was close to $f_{0}$ $\left(R 1: f_{0}\right.$ tuning) and $\mathrm{R} 2$ was close to $2 f_{0}\left(R 2: 2 f_{0}\right.$ tuning) for the measured data on altos and sopranos.

It can be seen from Fig. 6 that all sopranos employed $R 1: f_{0}$ tuning over some part of their range for every vowel studied. Their measured value of $R 1$ for speech was usually within, or close to, this tuning range. Consequently the lower value of $R 1$

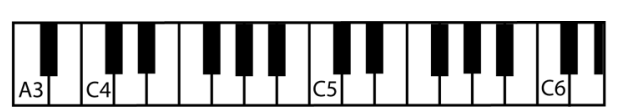

'hard'
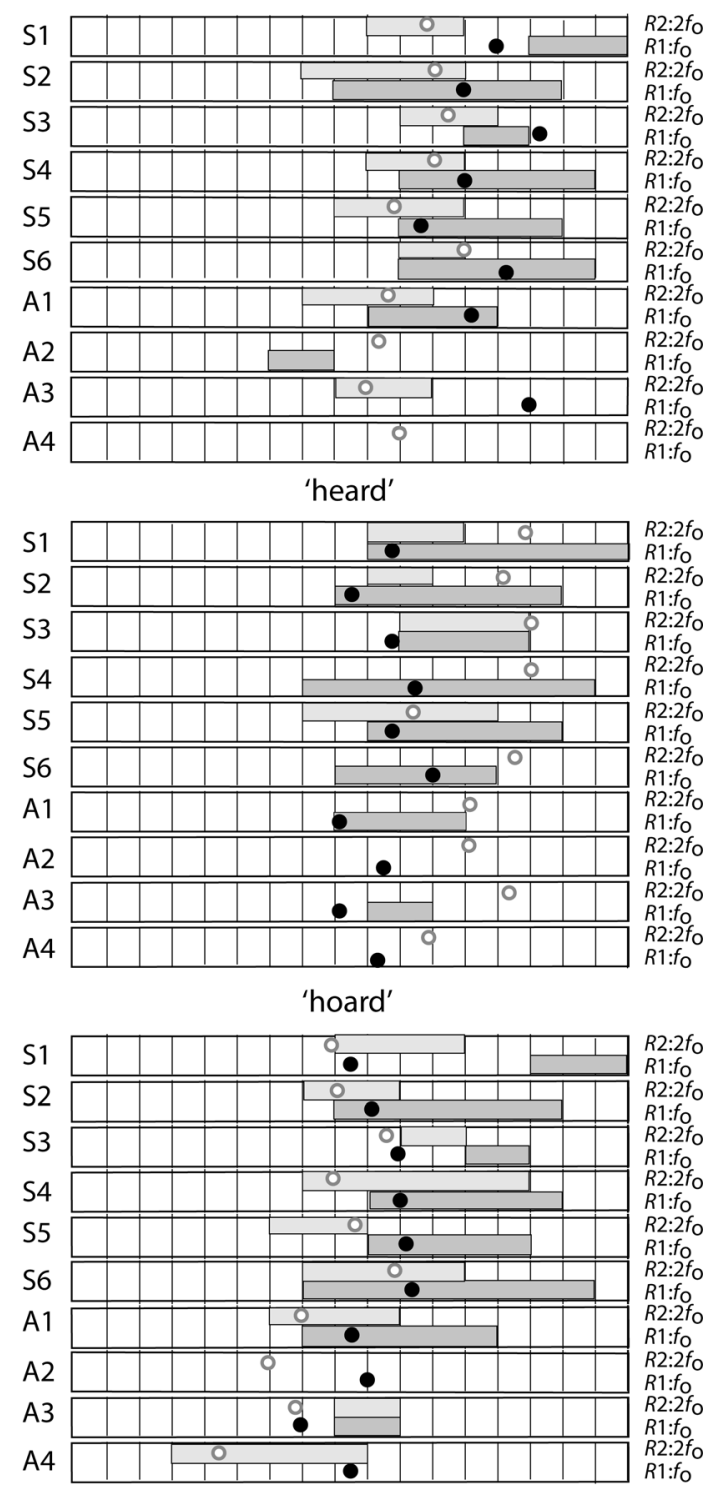

'who'd'

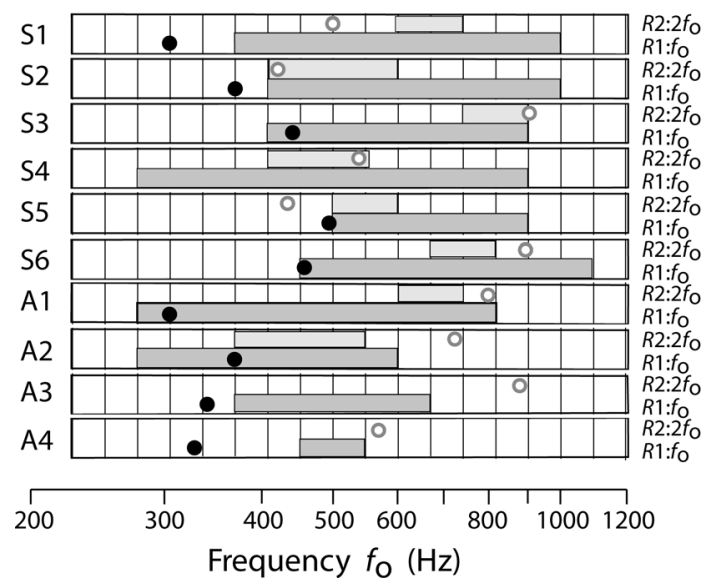

FIG. 6. The frequency ranges for resonance tuning for female singers measured for four different vowels. For each singer the lower darker shaded box indicates $R 1: f_{0}$ tuning and the upper lighter shaded box indicates $R 2: 2 f_{0}$ tuning. The full and open circles indicate the value for $R 1$ and $R 2 / 2$, respectively, measured for that singer for speech. 

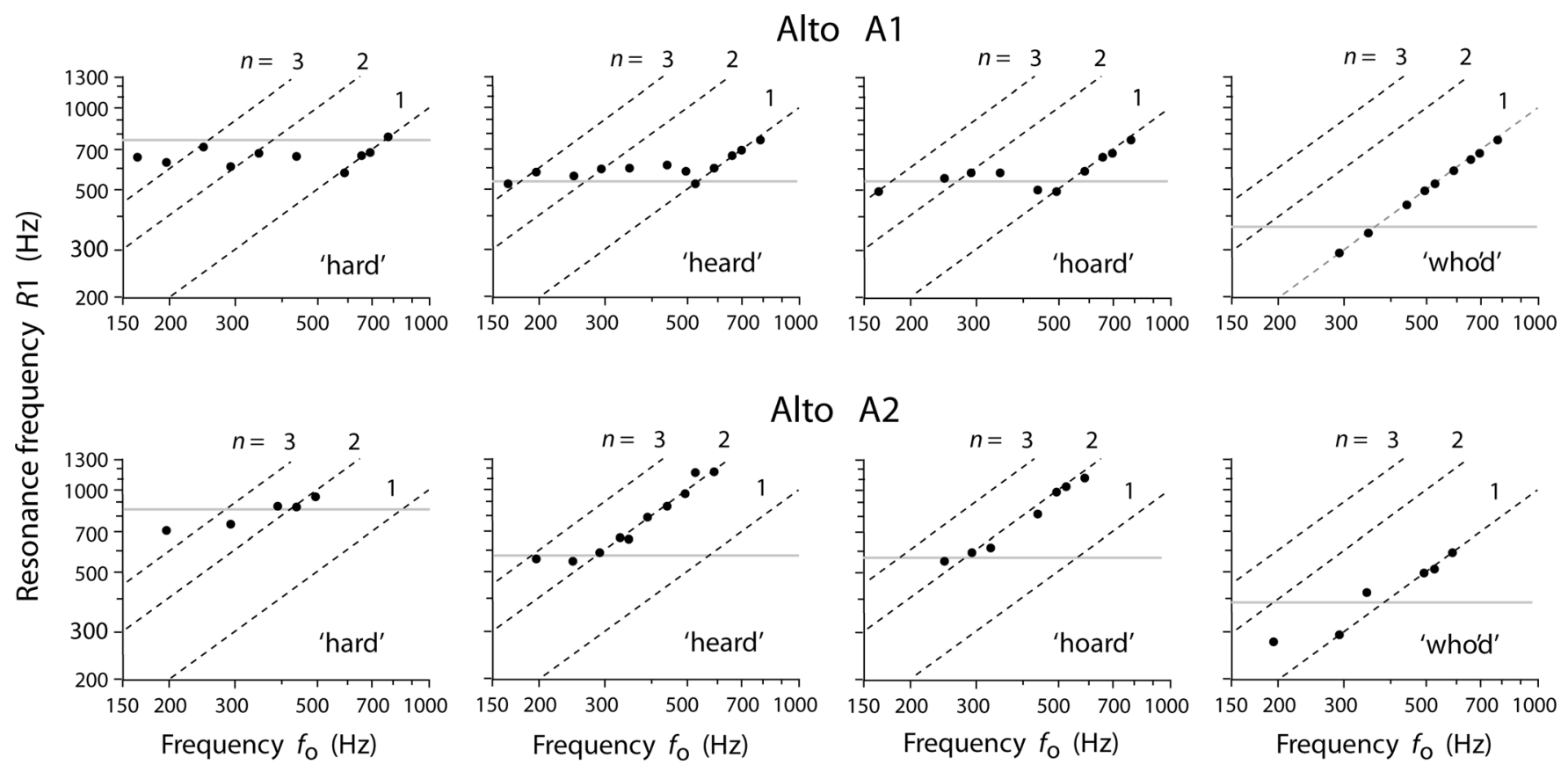

FIG. 7. Examples of two different tuning strategies used by altos. Alto A1 used systematic $R 1: f_{0}$ tuning once $f_{0}$ approached the value of $R 1$ measured in speech. Alto A2 used $R 1: f_{0}$ tuning for the vowel with the lowest value of $R 1$ in speech, but switched to $R 1: 2 f_{0}$ tuning for the other vowels. The horizontal lines indicate the values of $R 1$ measured for that singer and vowel in speech. In two cases (A1-who'd and A2-hard) measurements for speech were not available and consequently the average values for the other altos were used. The dashed diagonal lines indicate the relationships $R \mathrm{i}=n f_{0}$. The standard deviations in $R 1$ calculated across all vowels from measurements repeated at the same pitch were $\pm 2.1 \%$ and $\pm 7.7 \%$ for A1 and A2, respectively.

for "who'd" meant that $R 1: f_{0}$ tuning commenced at a lower pitch for this vowel and extended over a greater range. This is consistent with the results of Joliveau et al. (2004a,b).

Figure 6 also shows that most sopranos employ $R 2: 2 f_{0}$ tuning over at least a small part of their range, and this often occurs simultaneously with $R 1: f_{0}$ tuning (though not often for the vowel in "hoard"). This is not surprising: the most commonly observed gesture that increases $R 1$ is increasing mouth opening. Although $R 2$ depends primarily on tongue shape and position, it also increases with increasing mouth opening. Consequently, $R 2: 2 f_{0}$ tuning over part of the range of $R 1: f_{0}$ tuning might require little extra adjustment. Figure 4 shows an example of simultaneous $R 1: f_{0}$ and $R 2: 2 f_{0}$ tunings.

\section{Resonance tuning in the alto range}

Figure 7 shows examples of two different tuning strategies used by altos. Alto A1 used systematic $R 1: f_{0}$ tuning once $f_{0}$ became comparable with the value of $R 1$ measured in speech. Alto A2 used $R 1: f_{0}$ tuning for the vowel with the lowest value of $R 1$ in speech but switched to $R 1: 2 f_{0}$ tuning for the other vowels. This allowed resonance tuning to occur at lower pitches than if $R 1: f_{0}$ tuning were used.

\section{E. How closely are resonances tuned to one of the harmonics?}

The values of $\bar{\Delta}_{\mathrm{i}}$ and $\bar{\Delta}_{\mathrm{i}}^{\mathrm{o}}$ presented in Fig. 5 were calculated over the complete measured range for each singer and vowel. It is now interesting to examine how the resonance frequencies are distributed around the nearest harmonic. Accordingly Fig. 8 presents histograms of the frequency difference $R 1-f_{0}$ (i.e., $\Delta_{1}$ ). Figure 8 (a) presents the combined data for all the combinations of soprano and vowel measured in this study. A broad peak that is approximately $20 \mathrm{~Hz}$ wide and centred upon $R 1=f_{0}$ is apparent. Figure 8(b) presents the combined data for all the combinations of soprano and vowel measured in this laboratory: Six from this study, nine from Joliveau et al. (2004b) and 12 from Garnier et al. (2010), and a narrower peak when $R 1=f_{0}$ is now visible.
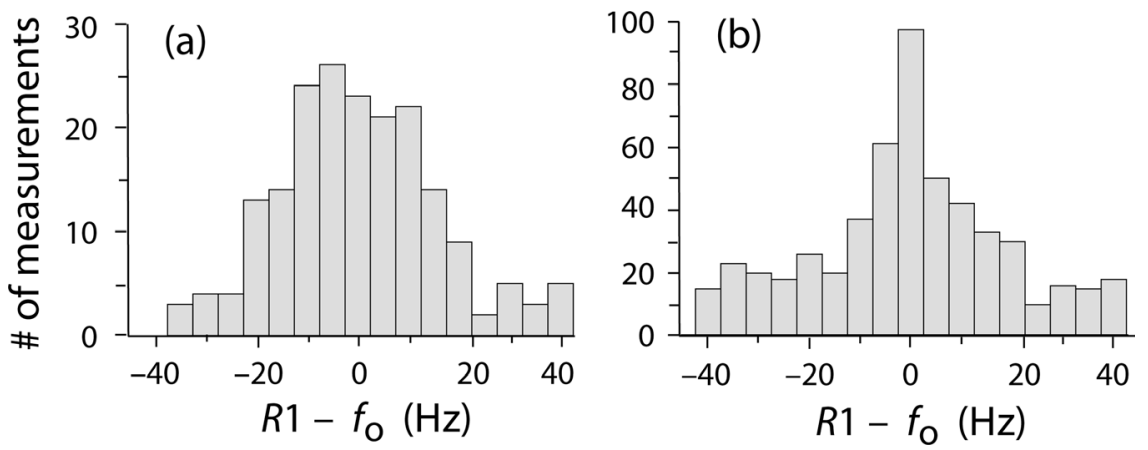

FIG. 8. Histograms showing the distribution of the measured values of $R 1$ about $f_{0}$. (a) presents the combined data for all the 190 combinations of soprano and vowel measured in this study. (b) presents the combined data for all the 511 combinations of soprano and vowel measured in this laboratory; six sopranos and four vowels from this study, nine sopranos and four vowels from Joliveau et al. (2004b) and 12 sopranos and one vowel from Garnier et al. (2010). 
These histograms suggest that, within the resolution of our measurements, sopranos appear to tune $R 1 \approx f_{0}$, rather than just tuning $R 1>f_{0}$.

Table $\mathrm{V}$ presents the values of $\bar{\Delta}_{\mathrm{i}}$ for the individual sopranos and altos calculated only for the measurements where resonance tuning was apparent. The histograms for $\Delta_{1}$ (Fig. 8) and $\Delta_{2}$ indicate that, at least for these experiments, resonance tuning could be assumed when $\Delta_{\mathrm{i}}<25 \mathrm{~Hz}$. Table $\mathrm{V}$ indicates that the average difference $\bar{\Delta}_{1}$ during resonance tuning is around $9 \mathrm{~Hz}$; this is similar to the resolution of our technique for measuring tract resonances. The average value of $\bar{\Delta}_{2}$ was around $12 \mathrm{~Hz}$.

One important consideration is whether $R \mathrm{i}$ is tuned higher or lower in frequency than the nearest harmonic, as this will make the acoustic load on the vocal folds caused by the vocal tract to be inertive or compliant, respectively.

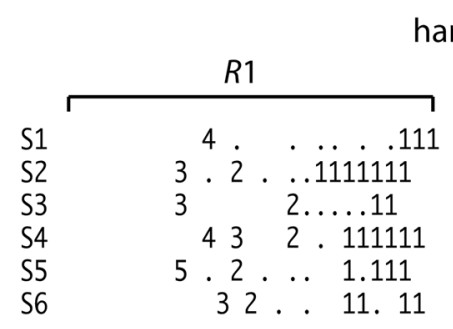

hard

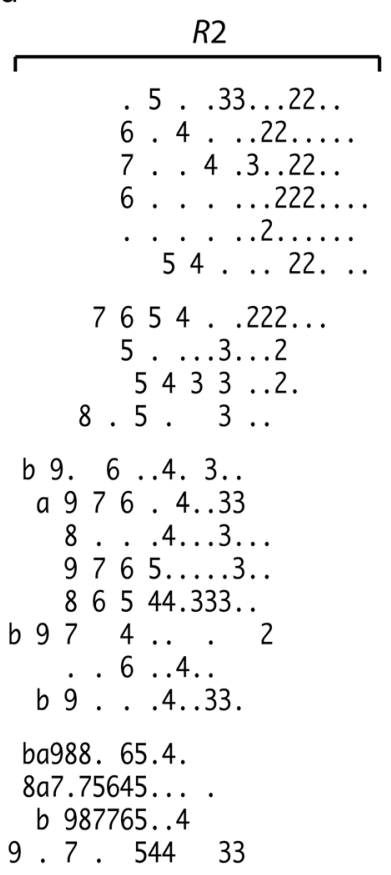

$$
\begin{aligned}
& 43322 \text {. } 1111 \\
& .322 . \\
& 6543
\end{aligned}
$$

665.32.

$65.4333 . .22$

$543.222 \ldots$

643.3 .222 .

33

$67.543 \ldots 22 \ldots \ldots 1$

T8 322 .

$\begin{array}{lll}\text { B1 } & 645534 & .22\end{array}$

B2 554443333222 .

B3 $6.44333 . .2$

B4 4.333 .
T7 4 . 222..

$$
1
$$

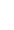

$$
\begin{aligned}
& \text {. } 5 . .33 \ldots 22 \ldots \\
& 6.4 . .22 \ldots \ldots \\
& 7 \text {. } 4.3 . .22 \ldots \\
& 6 \text {. . . . 2222... } \\
& 54 \ldots 2 \ldots
\end{aligned}
$$$$
654 \ldots .222 \ldots
$$$$
5 \ldots 3 \ldots 2
$$$$
5433 \ldots 2
$$

b $9.6 \ldots 4.3$.

\begin{tabular}{|c|c|c|}
\hline & & \\
\hline & $R 1$ & $R 2$ \\
\hline $\begin{array}{l}\text { S1 } \\
\text { S2 } \\
\text { S3 } \\
\text { S4 } \\
\text { S5 } \\
\text { S6 }\end{array}$ & 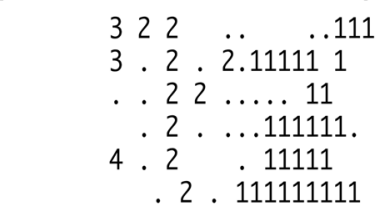 & $\begin{array}{c}54 . \ldots 2222 \ldots \\
5.333222 \ldots \\
. .4 .3 \ldots 22 \ldots \\
.4 .222 .22 \ldots \\
.4 .322 \ldots \ldots \\
.33222 \ldots \ldots\end{array}$ \\
\hline $\begin{array}{l}\text { A1 } \\
\text { A2 } \\
\text { A3 } \\
\text { A4 }\end{array}$ & 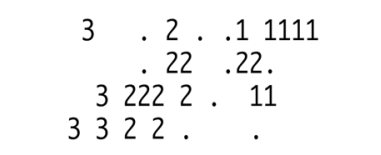 & $\begin{array}{l}5.3 \ldots \\
4 \cdot 33 \ldots \\
4.4 .4222 \ldots \\
54 \cdot .2222\end{array}$ \\
\hline $\begin{array}{l}\text { T1 } \\
\text { T2 } \\
\text { T3 } \\
\text { T4 } \\
\text { T5 } \\
\text { T6 } \\
\text { T7 } \\
\text { T8 }\end{array}$ & 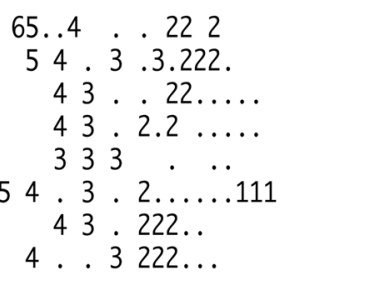 & 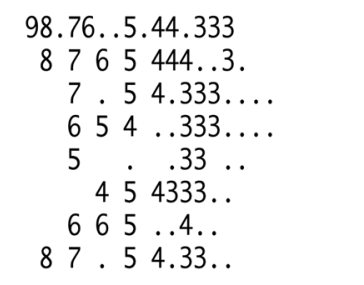 \\
\hline & 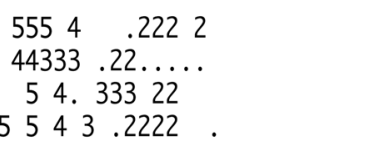 & $\begin{array}{c}a 98.7665444 \\
876.54 .33 \ldots \\
9876 \ldots 44 . \\
7765544 \ldots 3 \ldots\end{array}$ \\
\hline
\end{tabular}

a $976.4 . .33$

$8 \ldots 4 \ldots 3 \ldots$

\begin{tabular}{|c|c|c|}
\hline & $R 1$ & $R 2$ \\
\hline $\begin{array}{l}\text { S1 } \\
\text { S2 } \\
\text { S3 } \\
\text { S4 } \\
\text { S5 } \\
\text { S6 }\end{array}$ & 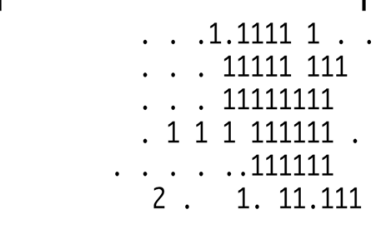 & $\begin{array}{c}.4 \ldots 2 \ldots \\
.3 .2222 \ldots \ldots \\
. .5 \ldots 22 \\
5.22 \ldots 2 \ldots \\
. .2 \ldots 22 \ldots \ldots \\
54.3 \ldots 22 \ldots\end{array}$ \\
\hline $\begin{array}{l}\text { A1 } \\
\text { A2 } \\
\text { A3 } \\
\text { A4 }\end{array}$ & 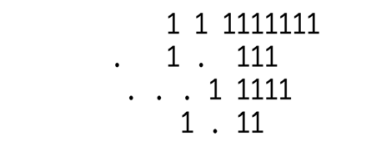 & 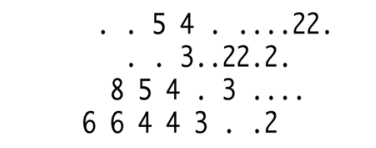 \\
\hline $\begin{array}{l}\text { T1 } \\
\text { T2 } \\
\text { T3 } \\
\text { T4 } \\
\text { T5 } \\
\text { T6 } \\
\text { T7 } \\
\text { T8 }\end{array}$ & 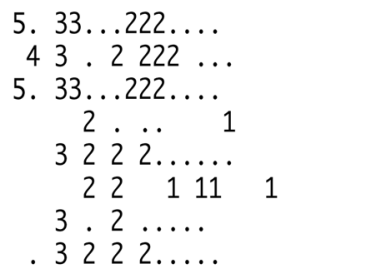 & 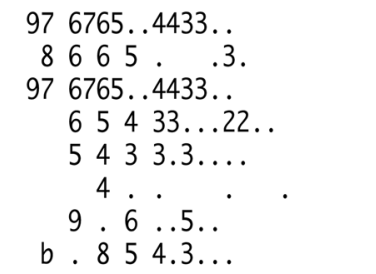 \\
\hline $\begin{array}{l}\text { B1 } \\
\text { B2 } \\
\text { B3 } \\
\text { B4 }\end{array}$ & $\begin{array}{cc}4 & 2 \ldots \\
332 & 2 \ldots 1.1 .1 \\
3 & 33 \ldots 2222 \\
4 & .3 \ldots 2 \ldots\end{array}$ & $\begin{array}{c}9 \ldots 555444333 \\
888856.54 \ldots 3 \ldots \\
\text { e b98..66.5 } \\
996 \quad .544 .33 \ldots\end{array}$ \\
\hline & 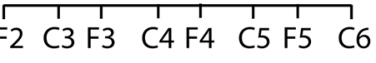 & F2 C3 F3 C4F4 C5 F5 C \\
\hline
\end{tabular}

$9765 \ldots . .3$.

.. $6 . .4$

b $987765 . .4$

9.7.544 33

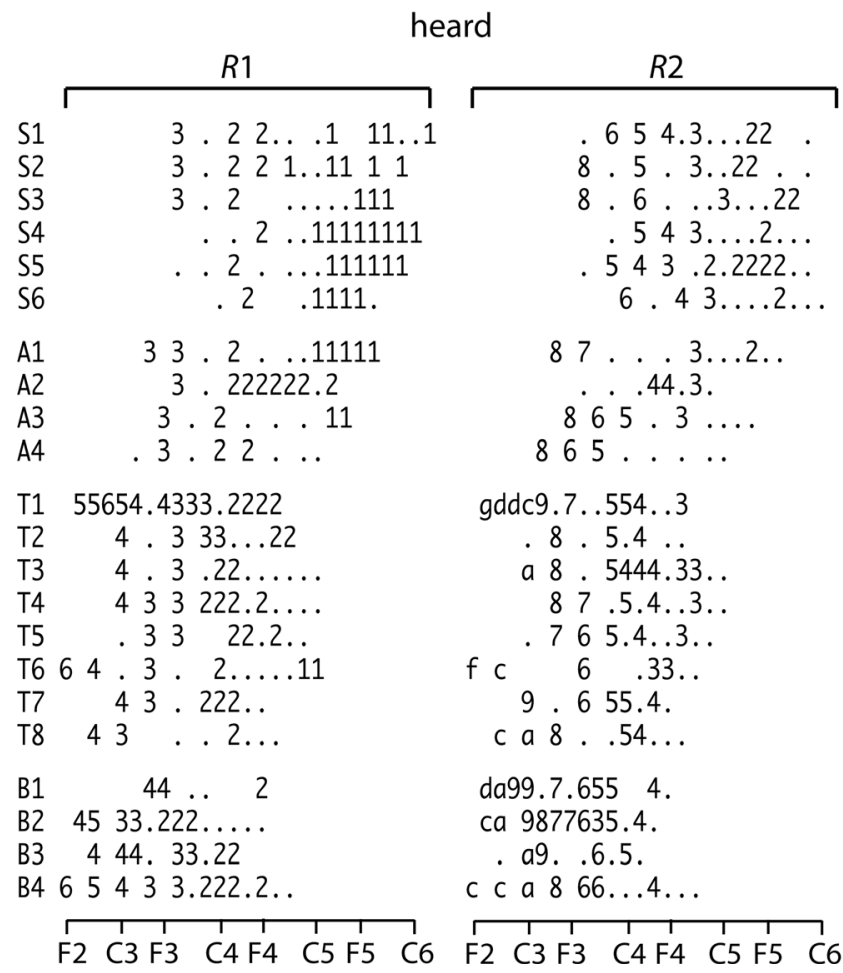

who'd

FIG. 9. The proximity of the resonances $R 1$ and $R 2$ to the $n$th harmonic of the sung pitch $f_{0}$. The numbers in the figure indicate the value of $n$ for the harmonic that was nearest to the resonance, provided the frequency difference $\bar{\Delta}_{\mathrm{i}}$ was $<50 \mathrm{~Hz}$. A dot indicates the measurement where the frequency difference between harmonic and resonance exceeded $50 \mathrm{~Hz}$. Harmonics greater than the ninth are indicated using the notation; $\mathrm{a}=10, \mathrm{~b}=11, \mathrm{c}=12, \mathrm{~d}=13, \mathrm{e}=14, \mathrm{f}=15$, and $\mathrm{g}=16$. 
TABLE V. The average frequency differences between resonance $R \mathrm{i}$ and the closest harmonic for altos and sopranos calculated over the regions where $R 1: f_{0}$ and $R 2: 2 f_{0}$ tunings occurred. The symbol $\bar{\Delta}_{\mathrm{i}}$ indicates the average absolute difference and $\bar{\delta}_{i}$ indicates the average signed difference from the $n$th harmonic. The number of measurements for which $R \mathrm{i} \geq n f_{0}$ and $R \mathrm{i}<n f_{0}$ are indicated by $m_{+}$and $m_{-}$, respectively.

\begin{tabular}{lccccccc}
\hline \hline & \multicolumn{3}{c}{$R 1: f_{0}$ tuning } & & \multicolumn{3}{c}{$R 2: 2 f_{0}$ tuning } \\
\cline { 2 - 4 } \cline { 6 - 7 } Singer & $\bar{\Delta}_{1}(\mathrm{~Hz})$ & $\bar{\delta}_{1}(\mathrm{~Hz})$ & $m_{+} / m_{-}$ & & $\bar{\Delta}_{2}(\mathrm{~Hz})$ & $\bar{\delta}_{2}(\mathrm{~Hz})$ & $m_{+} / m_{-}$ \\
\hline S1 & 9 & 2 & $6 / 8$ & & 11 & -6 & $2 / 6$ \\
S2 & 8 & -1 & $8 / 11$ & & 11 & -3 & $2 / 2$ \\
S3 & 9 & -4 & $3 / 3$ & & 10 & 3 & $3 / 3$ \\
S4 & 12 & -4 & $10 / 13$ & & 11 & 8 & $5 / 2$ \\
S5 & 9 & -5 & $6 / 14$ & & 10 & 0 & $2 / 3$ \\
S6 & 11 & 10 & $17 / 2$ & & 13 & 11 & $6 / 1$ \\
A1 & 9 & -6 & $6 / 16$ & & 13 & 0 & $2 / 5$ \\
A3 & 9 & -6 & $2 / 7$ & - & - & - \\
All & 9 & -2 & $60 / 80$ & 12 & 4 & $23 / 22$ \\
\hline \hline
\end{tabular}

Models suggest that this has implications for the amplitude and stability of vocal fold vibration (Titze, 1988; Fletcher, 1993). To investigate this, the following three new variables are introduced,

$\bar{\delta}_{i}=$ the average signed difference $\left(R \mathrm{i}\left(f_{0}\right)-n f_{0}\right)$

$m_{+}=$number of measurements in which $\left(R \mathrm{i}\left(f_{0}\right)>n f_{0}\right)$

$m_{-}=$number of measurements in which $\left(R \mathrm{i}\left(f_{0}\right)<n f_{0}\right)$

where $n$ denotes the nearest harmonic. Table $\mathrm{V}$ shows that the values for $\bar{\delta}_{1}$ tend to be slightly negative, suggesting that $R 1\left(f_{0}\right)<n f_{0}$ for most singers. This is also supported by the ratio $m_{+} / m_{-}$which was usually $\leq 1$. However, for $R 2$ the ratio $m_{+} / m_{-}$was typically around 1 , indicating that $R 2$ was equally likely to be above or below $2 f_{0}$ in frequency. The exception was S6, where the values of $\bar{\delta}_{1}$ and $\bar{\delta}_{2}$ indicate that $R 1$ was tuned around $10 \mathrm{~Hz}$ above $f_{0}$, and $R 2$ was tuned around $11 \mathrm{~Hz}$ above $2 f_{0}$, for almost all measurements. It should be remembered that these measured values of the differences $\bar{\Delta}_{\mathrm{i}}$ and $\bar{\delta}_{i}$ are of the same order as the resolution of our technique, and it would only require a small systematic error in our estimates of the resonance frequencies to skew their distribution about the closest harmonic.

\section{F. Resonance tuning by tenors and baritones}

Although Fig. 1 illustrates that many possible tuning strategies exist for the lower pitched voice, Fig. 5 indicates that significant improvements in matching resonances to harmonics are much less common than for the higher pitched voices. To help identify tuning strategies, and also to provide comparison with the strategies used at higher pitch, Fig. 9 shows the nearest harmonic to each $R$ i provided that $\Delta_{\mathrm{i}}<50$ $\mathrm{Hz}$. Thus the numeral 1 appearing in the $R 1$ region of the figure indicates that $R 1: f_{0}$ tuning within $50 \mathrm{~Hz}$ was evident at this pitch for this singer and vowel, and a numeral 3 in the $R 2$ region would indicate $R 2: 3 f_{0}$ tuning at that pitch.

Figure 1 shows that the $R 1: f_{0}$ tuning used by all sopranos, and some altos, is possible for the higher range of the tenor and baritone voice. However Fig. 9 shows that it was only used sparingly by one tenor in our sample, with the greatest range being for the vowel with the lowest value of $R 1$ in speech (who'd) — see Fig. 10.

Figure 1 indicates that $R 1: 2 f_{0}$ and $R 1: 3 f_{0}$ tunings are possible in the range below about $\mathrm{C} 5$, and most of the singers used these tunings, even if for only a small part of their range-see Fig. 10. Figure 10 also provides an example where tenor T1 successively exhibited $R 1: 4 f_{0}, R 1: 3 f_{0}$ and $R 1: 2 f_{0}$ tunings once the appropriate harmonic approached the value of $R 1$ in speech as the pitch increased. Tunings such as shown in Fig. 10 cannot be maintained over a wide pitch range because the value

Tenor T6
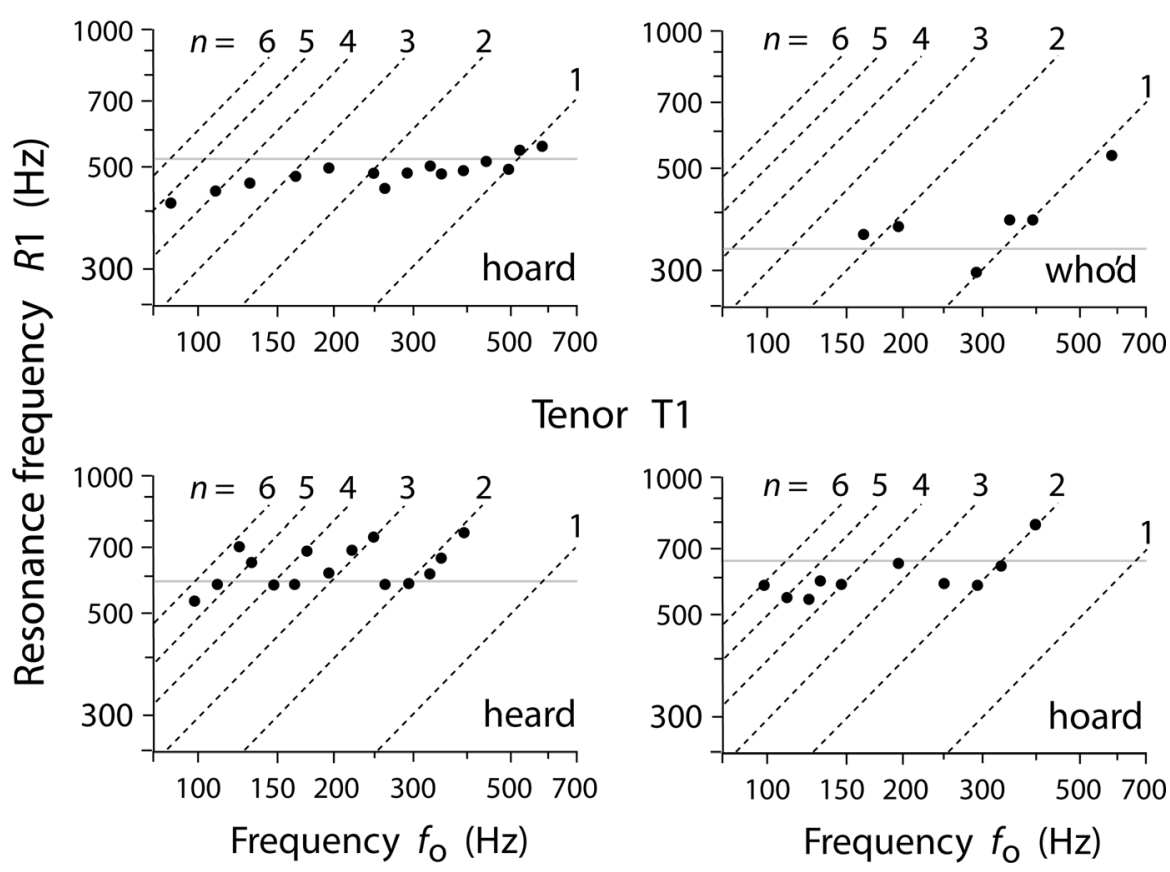

FIG. 10. An example showing some different tunings of $R 1$ used by tenors. Tenor T6 is seen to use $R 1: f_{0}$ tuning once the pitch approaches the value of $R 1$ in speech. Tenor T1 used $R 1: 2 f_{0}$ tuning once $2 f_{0}$ approached the value of $R 1$ in speech. For the vowel in "heard," T1 also exhibited $R 1: 3 f_{0}$ and $R 1: 4 f_{0}$ tunings when the relevant harmonic approached the value of $R 1$ in speech. The horizontal lines indicate the values of $R 1$ measured for speech for that singer and vowel. The standard deviations in $R 1$ calculated from measurements repeated at the same pitch for the vowels shown were $\pm 4.0 \%$ and $\pm 3.5 \%$ for $\mathrm{T} 1$ and $\mathrm{T} 6$, respectively. 


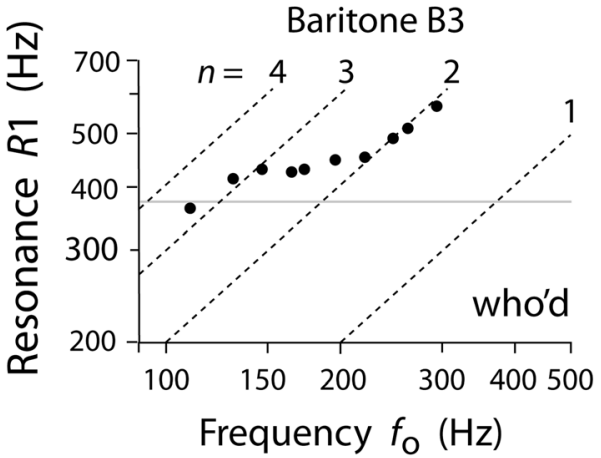

of $R 1$ rapidly approaches the upper limit of its range. Tenors often exhibited $R 1$ within $50 \mathrm{~Hz}$ of harmonics above the fourth, although only for one or two notes. Figure 11 shows an example of $R 1: 2 f_{0}$ tuning by baritone B3. Figure 11 also shows an interesting example where baritone B2 increases $R 1$ systematically with increasing pitch, but maintains $R 1$ just above $f_{0}$ so $R 1 \approx 1.15 f_{0}$. One possible explanation is that the bandwidth of $R 1$ in this case was such that these values of $\Delta_{1}$ might still produce a useful increase in volume.

For some singers and vowels there appeared also to be deliberate tuning of $R 2$. Figure 12 shows how, as the pitch increases, baritone $\mathrm{B} 1$ successively uses $R 2: 5 f_{0}, R 2: 4 f_{0}$, and $R 2: 3 f_{0}$ tunings.

\section{G. Consequences for singing synthesis-by-rule}

These measurements have some implications for synthesis-by-rule singing systems. One main observation is that $R 1$ should not be kept constant at the value for speech, but should be varied with pitch, even in cases where there is no tuning of resonances to harmonics. It presumably reflects the adjustments in articulation that commonly accompany a pitch rise, such as mouth opening, jaw lowering, and larynx rise. The systematic $R 1: f_{0}$ tuning observed for female singers, when the pitch approaches the normal range of $R 1$, should be implemented as a main rule. The tuning of $R 1$ to $2 f_{0}$ or higher harmonics could be implemented as an additional, but not compulsory, rule. Its perceptual effect on

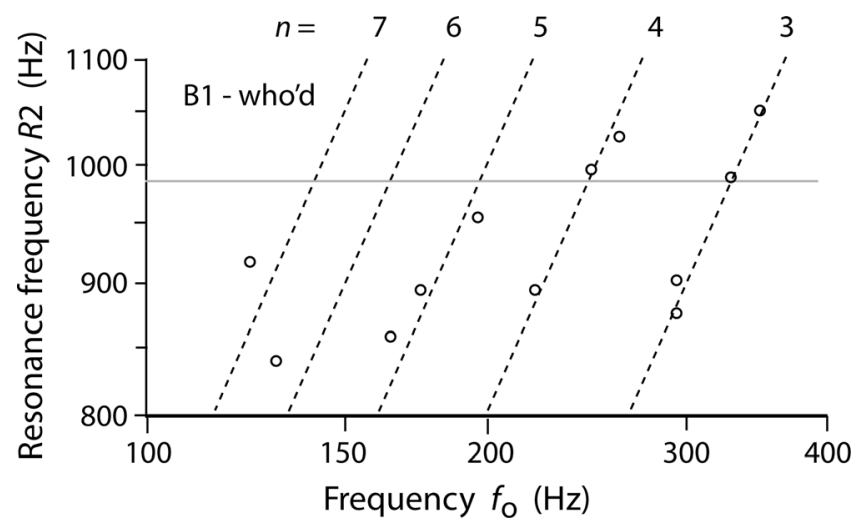

FIG. 12. An example showing three different tunings of $R 2$ used by baritone B1 for the vowel in "who'd." As the pitch increases the singer successively uses $R 2: 5 f_{0}, R 2: 4 f_{0}$, and $R 2: 3 f_{0}$ tuning. The horizontal line indicates the value of $R 2$ measured for speech for this singer. The standard deviation in $R 2$ calculated from repeated measurements repeated at the same pitch for this singer and vowel was $\pm 1.1 \%$.
Baritone B2

432

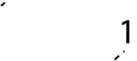

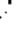

FIG. 11. An example showing different tunings of $R 1$ used by baritones for the vowel in "who'd." Baritone B3 is seen to use $R 1: 2 f_{0}$ tuning once the pitch exceeds G3 ( $f_{0}$ $\sim 200 \mathrm{~Hz}$ ). Baritone B2 provides a rare example where $R 1$ is consistently and clearly tuned above $f_{0}$. The horizontal lines indicate the values of $R 1$ measured for speech for that singer and vowel. The standard deviations in $R 1$ calculated from repeated measurements repeated at the same pitch for the vowel in "who'd" were $\pm 3.5 \%$ and $\pm 9.0 \%$ for $\mathrm{B} 2$ and $\mathrm{B} 3$, respectively. timbre lies beyond the scope of the present study, but has been mentioned in previous works (Boersma and Kovacic, 2006; Henrich et al., 2007). The R2:2 $f_{0}$ tuning observed here, and also reported by Garnier et al. (2010), could also be implemented as a possible rule in the top range of soprano voice.

\section{CONCLUSIONS}

This study confirms and extends the occurrence of $R 1: f_{0}$ tuning by sopranos in the higher part of their range. All of the 27 sopranos studied in this laboratory [six in this study, nine in Joliveau et al. (2004b) and 12 in Garnier et al. (2010)] have shown $R 1: f_{0}$ tuning over at least part of their upper range - a total of 72 separate singer/vowel combinations. Such consistency might encourage composers and librettists to consider matching vowel to pitch when writing for the soprano voice (Smith and Wolfe, 2009).

Most sopranos employed $R 2: 2 f_{0}$ tuning over at least a part of their range, and this often occurred simultaneously with $R 1: f_{0}$ tuning.

Altos use $R 1: f_{0}$ tuning for the vowels with lower values of $R 1$ in speech. They may switch to $R 1: 2 f_{0}$ tuning in lower part of their range.

Tenors and baritones generally used $R 1: 2 f_{0}$ and $R 1: 3 f_{0}$ tunings over at least part of their range. A number of different tunings to higher harmonics occurred at lower pitch. Occasionally a detailed resonance tuning of $R 2$ occurred.

The implications for singing synthesis are that $R 1: f_{0}$ tuning should be implemented for sopranos as a necessary rule, and that the tuning of $R 1$ to $2 f_{0}$ or higher harmonics could be implemented as an additional, but not compulsory, rule.

The results indicate that singers can repeatedly tune their resonances with a precision of typically $20 \mathrm{~Hz}$.

There can be considerable differences in the resonance strategies used by singers, particularly for voices in the lower ranges. Care should consequently be taken in extrapolating results from a single subject.

\section{ACKNOWLEDGMENTS}

We warmly thank our volunteer subjects for their patience and availability. We thank the Australian Research Council for support and Maëva Garnier for helpful discussions.

\footnotetext{
${ }^{1}$ In acoustics, the word "formant" is variously used to describe a broad peak in the spectral envelope, the acoustic resonance in a system that gives rise to it, or a property of a filter used to model the system. To avoid possible confusion "formant" is not used in this paper.
} 
Benolken, M., and Swanson, C. (1990). "The effect of pitch-related changes on the perception of sung vowels," J. Acoust. Soc. Am. 87, 1781-1785.

Boersma, P., and Kovacic, G. (2006). "Spectral characteristics of three styles of Croatian folk singing," J. Acoust. Soc. Am. 119, 1805-1816.

Bunch, M., and Chapman, J. (2000). "Taxonomy of singers used as subjects in research," J. Voice 14, 363-369.

Clark, J., Yallop, C., and Fletcher, J. (2007) An Introduction to Phonetics and Phonology, 3rd ed. (Basil Blackwell, Oxford), pp. 240-292.

Cleveland, T. F. (1977). "Acoustic properties of voice timbre types and their influence on voice classification,” J. Acoust. Soc. Am. 61, 1622-1629.

Dowd, A., Smith, J. R., and Wolfe, J. (1998). "Learning to pronounce vowel sounds in a foreign language using acoustic measurements of the vocal tract as feedback in real time," Lang. Speech 41, 1-20.

Epps, J., Smith, J. R., and Wolfe, J. (1997). "A novel instrument to measure acoustic resonances of the vocal tract during speech," Meas. Sci. Technol. 8, 1112-1121

Fant, G. (1970). Acoustic Theory of Speech Production (Mouton, Hague, The Netherlands), pp. 15-26.

Fletcher, N. H. (1993) "Autonomous vibration of simple pressure-controlled valves in gas flows," J. Acoust. Soc. Am. 93, 2172-2180.

Garnier, M., Henrich, N., Smith, J., and Wolfe, J. (2010). "Vocal tract adjustments in the high soprano range," J. Acoust. Soc. Am. 127, 3771-3780.

Henrich, N., Kiek, M., Smith, J., and Wolfe, J. (2007). "Resonance strategies used in Bulgarian women's singing style: A pilot study," Logoped. Phoniatr. Vocol. 32, 171-177.

Joliveau, E., Smith, J., and Wolfe, J. (2004a). "Tuning of vocal tract resonance by sopranos," Nature $\mathbf{4 2 7}, 116$

Joliveau, E., Smith, J., and Wolfe, J. (2004b). "Vocal tract resonances in singing: The soprano voice," J. Acoust. Soc. Am. 116, 2434-2439.

Miller, D. G., and Schutte, H. K. (1990). "Formant tuning in a professional baritone," J. Voice 4, 231-237.

Monsen, R. B., and Engebretson, A. M. (1983). "The accuracy of formant frequency measurements: A comparison of spectrographic analysis and linear prediction," J. Speech Hear. Res. 26, 89-97.

Music Acoustics (2010). www.phys.unsw.edu.au/jw/soprane.html (Last viewed October 14, 2010).
Neumann, K., Schunda, P., Hoth, S., and Euler, H. A. (2005). "The interplay between glottis and vocal tract during the male passaggio," Folia Phoniatr. Logop. 57, 308-327.

Schutte, H. K., and Miller, D. G. (1993). "Belting and pop, nonclassical approaches to the female middle voice: Some preliminary considerations," J. Voice 7, 142-150.

Schutte, H. K., Miller, D. G., and Duijnstee, M. (2005). "Resonance strategies revealed in recorded tenor high notes," Folia Phoniatr. Logop. 57, 292-307.

Scotto di Carlo, N., and Germain, A. (1985) "A perceptual study of the influence of pitch on the intelligibility of sung vowels," Phonetica 42, 188-197.

Smith, J. R. (1995). "Phasing of harmonic components to optimize measure signal-to-noise ratios of transfer functions," Meas. Sci. Technol. 6, 1343-1348.

Smith, J., and Wolfe, J. (2009). "Vowel-pitch matching in Wagner's operas: Implications for intelligibility and ease of singing," J. Acoust. Soc. Am. 125, EL196-EL201.

Stevens, K. N. (2000). Acoustic Phonetics, 1st ed. (MIT Press, Cambridge, MA), Current Studies in Linguistics Series, p. 30.

Sundberg, J. (1975). "Formant technique in a professional female singer," Acustica 32, 89-96.

Sundberg, J. (1987). The Science of the Singing Voice (Northern Illinois University Press, Dekalb, Illinois), pp. 124-129.

Sundberg, J., and Skoog, J. (1997). "Dependence of jaw opening on pitch and vowel in singers," J. Voice 11, 301-306.

Swerdlin, Y., Smith, J., and Wolfe, J. (2010). "The effect of whisper and creak vocal mechanisms on vocal tract resonances," J. Acoust. Soc. Am. 127, 2590-2598.

Titze, I. R. (1988). "The physics of small-amplitude oscillations of the vocal folds," J. Acoust. Soc. Am. 83, 1536-1552.

Titze, I. R. (2008). "Nonlinear source-filter coupling in phonation: Theory," J. Acoust. Soc. Am. 123, 2733-2749.

Titze, I. R., Mapes, S., and Story, B. (1994). "Acoustics of the tenor high voice," J. Acoust. Soc. Am. 95, 1133-1142.

Tom, K., and Titze, I. R. (2001). "Vocal intensity in falsetto phonation of a countertenor: An analysis by synthesis approach," J. Acoust. Soc. Am. 110, 1667-1676 